\section{The Multidimensional Prognostic Index (MPI) for the prognostic stratification of hospitalized older patients with COVID-19: a prospective multicenter observational cohort study. Objectives, study design and expected outcomes (MPI_COVID-19)}

\author{
Carlo Custodero, ${ }^{1}$ Alberto Cella, ${ }^{2}$ \\ Nicola Veronese, ${ }^{3}$ Margherita Azzini, ${ }^{4}$ \\ Filippo Fimognari, \\ Francesco Mattace-Raso, ${ }^{6}$ \\ Maria Cristina Polidori, ${ }^{7}$ Carlo Sabbà, ${ }^{1}$ \\ Alberto Pilotto ${ }^{1,2}$
}

${ }^{1}$ Clinica Medica Frugoni and Geriatric

Medicine, Department of

Interdisciplinary Medicine, University of

Bari Aldo Moro, Bari, Italy;

${ }^{2}$ Department Geriatric Care,

Orthogeriatrics and Rehabilitation,

Galliera Hospital, Genova, Italy;

${ }^{3}$ Azienda ULSS 3 Serenissima, Primary

Care Department, District 3, Venice,

Italy; ${ }^{4}$ Geriatrics Unit, Mater Salutis

Hospital, Legnago ULSS 9 Scaligera,

Verona, Italy; ${ }^{5}$ Geriatrics Unit, Azienda

Ospedaliera di Cosenza, Italy; ${ }^{6}$ Division

of Geriatrics, Department of Internal

Medicine, Erasmus MC University

Medical Center, Rotterdam, The

Netherlands; ${ }^{7}$ Department Medicine II,

University Hospital of Cologne, and

Cologne Excellence Cluster on Cellular

Stress Responses in Aging-associated

Diseases, CECAD, University of

Cologne, Cologne, Germany

\begin{abstract}
The emergent coronavirus-19 disease (COVID-19) pandemic posed and still poses serious issues in the management of the inpatients and in the resource allocation, in particular for those patients requiring Intensive Care Unit (ICU) management. Epidemiological data clearly suggest that multimorbid older patients have the poorest prognosis. However, it is conceivable that age and number of comorbidities alone do not reflect the real condition and the expected prognosis of the patients affected by COVID19. A different approach based on comprehensive geriatric assessment (CGA) could help to better identify older patients more at risk of dismal outcomes and who, at some
\end{abstract}

point of their clinical course, will need the ICU admission. The Multidimensional Prognostic Index (MPI) is a well-accepted tool derived from a standard CGA which allows to measure prognosis of older patients in different clinical settings including hospital. Therefore, we designed a multicenter, prospective, observational study to evaluate the role of MPI in predicting risk of ICU admission and in-hospital mortality among 500 COVID-19-positive older subjects admitted to geriatric and internal medicine wards. In addition, risk of re-hospitalization, institutionalization and death after 3 months from discharge will be assessed. The MPI yields a straightforward value from 0 to 1 and might be able to adequately stratify complex, vulnerable COVID-19 patients for best possible decision-making and treatment allocation.

\section{Introduction}

The recent diffusion of the coronavirus19 disease (COVID-19) has reached proportions to be declared a pandemic disease as it affected more than 100,000 persons in 100 different countries. ${ }^{1}$ According to recent epidemiological data from Italy, $96.3 \%$ of deceased patients are $>60$ years old and $87.7 \%$ is aged 70 years and older, showing that COVID-19 is a killer pathogen mainly in the aging population. ${ }^{2} \mathrm{~A}$ source of big concern among all the Health Systems is the number of hospital and intensive care beds available to host the increasing number of older patients who need intensive interventions.

A recent document from the Italian Society of Anesthesiology and Resuscitation (SIAARTI) proposes to allocate treatments according to life expectancy. ${ }^{3}$ Though the current guidelines did not suggest that age should be the only factor determining resource allocation, the committee acknowledged that crucial for the clinical decision could be the difficulty for the frail older patients to survive the prolonged intubation required to recover from COVID-19-related pneumonia. ${ }^{4}$

This points to the need to stratifying patients according their prognosis based upon validated criteria. Wynants and colleagues, in a metanalytic revision of the current literature, showed that proposed prediction models for prognosis of patients with COVID-19 infection lack of reliability and therefore could be more harmful than good. ${ }^{5}$ Besides age, sex and disease-specific determinants (e.g., computed tomography (CT) scans, inflammatory markers), ${ }^{5}$ it would be also important for the prognosis of older patients to assess functional, physical and
Correspondence: Carlo Custodero, Clinica Medica Frugoni and Geriatric Medicine, Department of Interdisciplinary Medicine, University of Bari Aldo Moro, P.zza Giulio Cesare 11, 70124, Bari, Italy.

E-mail: carlo.custodero@uniba.it,

Key words: MPI; COVID-19; prognosis; older adults.

Acknowledgments: this study is promoted by EuGMS (European Geriatric Medicine Society), Special Interest Group on Comprehensive Geriatric Assessment (SIG-on-CGA)

Contributions: AP conceived the study; $\mathrm{CC}$ wrote the manuscript; AC, NV, MA, FF, FM, $\mathrm{MCP}$ and $\mathrm{CS}$ were major contributors and critically revised the manuscript. All authors read and approved the final version of this manuscript.

Conflict of interests: the authors declare no potential conflict of interests.

Received for publication: 16 April 2020. Accepted for publication: 22 April 2020.

This work is licensed under a Creative Commons Attribution-NonCommercial 4.0 International License (CC BY-NC 4.0).

${ }^{\circ}$ Copyright: the Author(s), 2020

Licensee PAGEPress, Italy

Geriatric Care 2020; 6:9038

doi:10.4081/gc. 2020.9038

psycho-social factors that can be best addressed by multidimensional tools. ${ }^{6}$ The Multidimensional Prognostic Index (MPI) is a prognostic tool able to predict short-term and long-term mortality in older patients in different settings including hospital. MPI is calculated based on information derived from a standard Comprehensive Geriatric Assessment (CGA) exploring different domains such as functional status, cognitive performance, risk of pressure sores, nutrition, multi-morbidity, number of drugs and co-habitation status.

A consistent body of literature demonstrated that MPI has excellent accuracy and calibration in predicting length of hospital stay and clinical outcomes after hospitalization. ${ }^{8}$ Recently, results from the MPI_AGE European project reported that MPI was able to predict in-hospital mortality, as well as the risk of re-hospitalization, institutionalization, and access to home-care services. ${ }^{9}$ Nowadays, the MPI has been validated in over 54,000 older adults suffering from the most common chronic and acute age-related diseases associated with high mortality in over 50 international studies. ${ }^{10}$ It is consid- 
ered one of the most commonly used tools to ascertain presence and degree of frailty in hospital and primary care. ${ }^{11}$ Moreover, through the stratification of older patients in classes with different mortality risk, MPI has been shown to be an helpful instrument in the clinical decision making. ${ }^{10}$

Of note, previous studies confirmed the validity of MPI in predicting mortality of older patients hospitalized with pneumonia $^{12}$ and very recently, the MPI has been shown to predict the clinical outcome after non-invasive ventilation (NIV) in patients affected from respiratory insufficiency. ${ }^{13}$ However, there are no studies yet regarding the use of MPI to predict prognosis in older inpatients with COVID-19.

\section{Objectives}

\section{Main objective}

The main objective of the study is to evaluate in a population of hospitalized older patients affected by COVID-19, the reliability and accuracy of MPI in predicting: i) the admission to intensive care units (ICU) and ii) the risk of in-hospital mortality.

\section{Secondary objectives}

Secondary objectives of the study are to evaluate whether MPI could predict in this population: i) the length of stay, and the risk, over 3 months follow-up, of: ii) rehospitalization; iii) admission to long-term care facility; iv) mortality.

\section{Materials and Methods}

\section{Study design}

This is a multicenter, prospective, observational cohort study which will enroll hospitalized older patients positive for
COVID-19 from 10 centers across the European Union (EU). At baseline, all recruited patients will undergo data collection for the calculation of the CGA-based MPI. Clinical, biological and radiological parameters about COVID-19 infection will be collected. In addition, discharge allocation, therapy plan at discharge, length of hospital stay, main diagnosis and other diagnoses will be recorded. After 3 months from discharge, patients and/or caregivers will be contacted to collect information about survival and eventual re-hospitalizations/institutionalization.

\section{Sample size}

A recent multicenter study reported that hospitalized older subjects in MPI-3 high risk group had an in-hospital mortality rate of $6.4 \%$ (in subjects at low-risk in MPI-1 group the mortality rate was $0.7 \%) .{ }^{14}$ Based on these data, 328 patients are sufficient to demonstrate the primary outcome, i.e. inhospital mortality, with a first type error (alpha) of 5\% and a statistic power of $80 \%$. In this study we plan to recruit about 500 older hospitalized patients.

\section{Setting and participants}

This study will enroll a population of 500 hospitalized older patients ( $\geq 65$ years) consecutively admitted to geriatrics and internal medicine ward.

All the patients are required to have the following inclusion criteria: i) 65 or more years old; ii) confirmed diagnosis of COVID-19 through swab with RT-PCR; iii) willingness to participate to the study. Patients younger than 65 years or unwilling/unable to participate to the study will be excluded.

\section{Outcomes}

Primary outcomes are the ICU admission from geriatrics or internal medicine wards and the in-hospital mortality.
Furthermore, we will assess as secondary outcomes: the length of hospital stay and during follow-up, until 3 months from discharge: mortality, rehospitalization, longterm care admission.

\section{Procedures}

Table 1 shows the information that will be collected at hospital admission. All the enrolled patients, who will meet the inclusion/exclusion criteria, will sign informed consent and have a standard CGA in order to collect the following information: personal data, functional, cognitive, and nutritional status, risk of pressure sores, co-morbidity, number of drugs taken, co-habitation status. Clinical data about COVID-19 infection will be recorded including signs and symptoms, day of swab, day of diagnosis, findings from standard (e.g., circulating inflammatory markers, chest X-ray, thorax ultrasound) or specific (e.g., chest CT, bronchoscopy) diagnostic tests. We will also report the following data: need of NIV or invasive mechanical ventilation, delirium assessment, discharge allocation, drug therapy at admission and at discharge, length of hospitalization, main diagnosis and secondary diagnoses coded through the ICD9-CM system. At 3 months after discharge, the patient or his/her caregiver will be contacted by phone call to determine: survival/mortality (eventually the date and cause of death), number of re-hospitalizations and/or institutionalization (Table 1).

\section{Multidimensional Prognostic Index}

The MPI is a prognostic tool based on a standardized CGA which allows to predict short- and long-term mortality in older subjects. MPI includes data derived from the eight following domains: i) Activities of Daily Living (ADL) - 6 items which evaluate the patient's functional status in daily life activities; ${ }^{15}$ ii) Instrumental Activities of Daily Living (IADL) - 8 items assessing the

Table 1. Procedures at the two different time-points in the MPI_COVID-19 study.

\begin{tabular}{ll} 
Recruitment (at hospital admission) & Follow-up (3 months after discharge) \\
Informed consent; & Survival/mortality (eventually the date and cause of death); \\
Demographics data; & Number of re-hospitalizations; \\
\hline Standard CGA in order to calculate MPI; & Long-term care facility admission \\
Clinical parameters related to COVID-19 infection; & \\
\hline Delirium assessment; & \\
Need of NIV or invasive mechanical ventilation; & \\
\hline Main diagnosis and secondary diagnoses coded through the ICD9-CM system; & \\
\hline Drug therapy at admission and at discharge; & \\
\hline Total length of stay and discharge allocation (e.g., admission to ICU, institutionalization, home) & \\
\hline MPI, Multidimension Prognostic Index; CGA, comprehensive geriatric assessment; NIV, non-invasive ventilation; ICU, Intensive Care Unit.
\end{tabular}


level of independence in the instrumental activities of daily life; ${ }^{16}$ iii) Short Portable Mental Status Questionnaire (SPMSQ) composed of 10 questions which assessed the patient's cognitive status, in particular spatio-temporal and personal orientation, memory, attention and computation ability; ${ }^{17}$ iv) Exton-Smith Scale (ESS) composed of 5 items assessing the risk of developing pressure sores; ${ }^{18} \quad$ v) Mini-Nutritional Assessment-short form (MNA-SF) which consists of 6 items evaluating the patient's nutritional status through based on anthropometric, dietary and subjective data; $\left.{ }^{19} \mathrm{vi}\right)$ Cumulative Illness Rating Scale (CIRS) consisting of 14 items which allow to identify the pathologies present at the time of patient recruitment and their severity by calculating the comorbidity and severity index respectively; ${ }^{20}$ vii) number of drugs usually taken by the patient; viii) cohabitation status which identifies the patient's socio-housing condition. To each domain is assigned a score corresponding to a risk, respectively: low (value of 0 ); moderate (value of 0.5 ); high (value of 1) (see Table 2).

The MPI is expressed as a continuous value from 0.0 (lowest risk of mortality) to 1.0 (highest risk of mortality). Appropriate and validated cut-off have been calculated to identify three levels of mortality risk as follows: MPI-1: mild risk (MPI values from 0.0 to 0.33 ), MPI-2: moderate risk (MPI values from 0.34 to 0.66$)$, MPI-3: severe risk (MPI values from 0.67 to 1.0 ). The calculation of the final MPI will be made through the software application available at the http://www.mpiage.eu.

\section{Clinical evaluation}

In addition, other clinical parameters related to the COVID-19 infection will be evaluated at hospital admission: body tem- perature (measured in $\mathrm{C}^{\circ}$ ), presence of cough, dyspnoea, diarrhea, parameters from blood gas analysis [i.e., $\mathrm{pH}, \mathrm{pO}_{2}, \mathrm{SpO}_{2}$, $\mathrm{pCO}_{2}, \quad \mathrm{HCO}_{3}^{-}, \quad \mathrm{pO}_{2} \quad(\mathrm{mmHg}) / \mathrm{O}_{2}$ Flux (L/min)], serum biomarkers (white blood cells, count of lymphocytes, C-reactive protein, $\mathrm{LDH}$ ), findings from imaging techniques (e.g. chest x-ray, thorax ultrasound, chest CT). Given the several risk factors for delirium development in these patients (e.g., age, sepsis, distance from caregivers), ${ }^{21}$ we will assess presence of this condition using the four 'A's Test (4AT), a screening tool for delirium which evaluates alertness, memory, attention and acute change or fluctuation in mental status. ${ }^{22}$ Finally, need of NIV or invasive mechanical ventilation, main diagnosis (and secondary diagnoses) at discharge in accordance with the ICD9-CM and drug therapy at discharge will be recorded.

\section{Data management and conservation of documentations}

The data will be managed at the E.O. Galliera Hospital - National Relevance and High Specialization Hospital, Genoa, Italy, coordinator center of the MPI_COVID-19 Project, which is designated as the center responsible for the processing and storing of personal data described above, in accordance with the rules of privacy and agreement to what is reported in this study protocol. All data will be collected in an electronic database in every center and transmitted anonymously to the coordinator center using standardized secure transmission rule in order to guarantee anonymity and protection of data.

\section{Statistical analysis}

General characteristics will be reported as frequencies (percentages) for categorical variables and mean \pm standard deviation for continuous variables. Comparisons across MPI categories will be performed using the Kruskal-Wallis test for singly ordered contingency tables, and linear by linear association test, for categorical and continuous variables, respectively.

Logistic binary regression analysis will be run, taking MPI at admission (in categories or as increase in 0.1 points) as exposure and admission to ICU, in-hospital mortality, and post-discharge (3-months) rehospitalization, institutionalization and mortality as outcomes. The strength of the association between MPI at admission and these outcomes will be reported as odds ratios (ORs) with 95\% confidence intervals (CIs), adjusted for age, gender and center. We will also calculate the adjusted risk that come from the adjusted predicted probability of the outcome of interest for each level of MPI, when the other covariates in the model are at their means. For all the outcomes, receiver operator characteristic curves will be analyzed to compare the sensitivity and specificity of MPI in predicting these negative outcomes, measuring the relative area under the curve with the correspondent 95\% CI. Two-sided alternatives with a significance level $(\mathrm{P}=0.05)$ will be considered for all the tests.

\section{Monitoring and quality assurance}

All partner centers will perform quality control and assurance procedures according to their internal standards and procedures. At least one technical meeting with all the centers involved in the data collection will be organized prior to the starting of the patient recruitment phase to ensure a proper implementation of the study. Moreover, at the start-up of the project the responsible for every center will conduct a meeting in their respective unit to share and adequately

Table 2. Ranges of values for each domain corresponding to the three risk levels in the Multidimensional Prognostic Index.

MPI

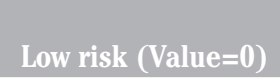

High risk (Value=1)

\begin{tabular}{llccc} 
1. & ADL & $6-5$ & $4-3$ & $2-0$ \\
2. & IADL & $8-6$ & $5-4$ & $3-0$ \\
\hline 3. & SPMSQ & $0-3$ & $4-7$ & $8-10$ \\
4. & ESS & $16-20$ & $10-15$ & $5-9$ \\
\hline 5. & MNA-SF & $12-14$ & $8-11$ & $0-7$ \\
6. & CIRS & 0 & $1-2$ & $\geq 3$ \\
\hline 7. & Number of drugs & $0-3$ & $4-6$ & $\geq 7$ \\
8. & Cohabitation status & Family & Nursing home & Alone \\
\hline Add the values of the single items and divide by 8 & & TOTAL SCORE MPI & \\
\hline
\end{tabular}

MPI, Multidimensional Prognostic Index; ADL, Activities of Daily Living; IADL, Instrumental Activities of Daily Living; SPMSQ, Short Portable Mental Status Questionnaire; ESS, Exton-Smith Scale; MNA-SF, Mini-Nutritional Assessment Short Form; CIRS, Cumulative Illness Rating Scale. 
inform the medical and nursing personnel participating to the project about the procedures for data collection. The same responsible will monitor, during the study, the personnel adherence to the collection protocol.

\section{Ethical issues}

The study will be conducted in accordance with the recommendations adopted by the $18^{\text {th }}$ World Medical Assembly, Helsinki 1964 and subsequent revisions and following Good Clinical Practices.

\section{Conclusions}

This study will provide new insights on the short and long-term prognosis of older adults affected by COVID-19. Criteria merely based on age and number of comorbidities might be not such really sensitive in the early identification of healthcare needs of older patients with COVID-19. We hypothesize that a single numerical value derived from a standard CGA, as the MPI score, could help the clinicians to better identify those patients more at risk of negative outcomes and requiring more intensive health support.

\section{References}

1. World Health Organization (WHO). Coronavirus disease 2019 (COVID-19): situation report, $45 ; 2020$.

2. Onder G, Rezza G, Brusaferro S. Casefatality rate and characteristics of patients dying in relation to COVID-19 in Italy. JAMA 2020 [Epub ahead of print].

3. Società Italiana di Anestesia Analgesia Rianimazione e Terapia Intensiva (SIARTI). Clinical ethics recommendations for the allocation of intensive care treatments, in exceptional, resourcelimited circumstances. Available from: http://www.siaarti.it/News/comunicato $\% 20$ raccomandazioni $\% 20$ di $\% 20$ etica $\% 20$ clinica $\% 20$ siaarti.aspx
4. Rosenbaum L. Facing Covid-19 in Italy - Ethics, logistics, and therapeutics on the epidemic's front fine. New Engl J Med 2020 [Epub ahead of print].

5. Wynants L, Van Calster B, Bonten MMJ, et al. Prediction models for diagnosis and prognosis of covid-19 infection: systematic review and critical appraisal. BMJ 2020;369:m1328.

6. Polidori MC, Maggi S, Mattace-Raso F, Pilotto A. The unavoidable costs of frailty: a geriatric perspective in the time of COVID-19. Geriatric Care 2020;6:8989.

7. Pilotto A, Ferrucci L, Franceschi M, et al. Development and validation of a multidimensional prognostic index for one-year mortality from comprehensive geriatric assessment in hospitalized older patients. Rejuvenation Res 2008;11:151-61.

8. Volpato S, Bazzano S, Fontana A, et al. on behalf of the MPI_Tri-Veneto Study Group. Multidimensional Prognostic Index predicts mortality and length of stay during hospitalization in the older patients: a multicenter prospective study. J Gerontol A Biol Sci Med Sci 2015;70:325-31.

9. Cruz-Jentoft AJ, Daragjati J, Fratiglioni L, et al. on behalf of the MPI AGE Investigators. Using the Multidimensional Prognostic Index (MPI) to improve cost-effectiveness of interventions in multimorbid frail older persons: results and final recommendations from the MPI_AGE European Project. Aging Clin Exp Res 2020 [Epub ahead of print]..

10. Pilotto A, Custodero C, Maggi S, et al. A multidimensional approach to frailty in older people. Ageing Res Rev 2020;60:101047. [Epub ahead of print].

11. Dent E, Martin FC, Bergman H, et al. Management of frailty: opportunities, challenges, and future directions. Lancet 2019;394:1376-86.

12. Pilotto A, Addante F, Ferrucci L, et al. The multidimensional prognostic index predicts short- and long-term mortality in hospitalized geriatric patients with pneumonia. J Gerontol A Biol Sci Med Sci 2009;64:880-7.
13. Gandolfo F, Corradi F, Custureri R, et al. Multidimensional Prognostic Index (MPI) predicts non-invasive mechanical ventilation (NIV) success and mortality in older people with acute hypoxemic respiratory failure (AHRF). Eur Geriatr Med 2018;9:P-572, S227.

14. Pilotto A, Veronese N, Daragjati J, et al.; on behalf of the MPI_AGE Investigators. Using - the Multidimensional Prognostic Index to predict clinical outcomes of hospitalized older persons: a prospective, multicentre, international study. J Gerontol A Biol Sci Med Sci 2019;74:1643-9.

15. Katz S, Downs TD, Cash HR, Grotz RC. Progress in development of the index of ADL. Gerontologist 1970;10: 20-30.

16. Lawton M, Brody E. Assessment of older people: self-maintaining and instrumental activities of daily living. Gerontologist 1969;9:179-86.

17. Pfeiffer E. A short portable mental status questionnaire for the assessment of organic brain deficit in elderly patients. J Am Geriatr Soc 1975;23:433-41.

18. Bliss MR, McLaren R, Exton-Smith AN. Mattresses for preventing pressure sores in geriatric patients. Monthly Bulletin of the Ministry of Health and the Public Health Laboratory Service 1966;25:238.

19. Kaiser MJ, Bauer JM, Ramsch C, et al. Validation of the Mini Nutritional Assessment Short-Form (MNA ${ }^{\circledR}-\mathrm{SF}$ ): A practical tool for identification of nutritional status. JNHA-J Nutr Health Aging 2009; 13:782.

20. Linn BS, Linn MW, Gurel L. Cumulative illness rating scale. J Am Geriatr Soc 1968;16:622-6.

21. LaHue SC, James TC, Newman JC, et al. Collaborative delirium prevention in the age of COVID-19. J Am Geriatr Soc 2020 [Epub ahead of print].

22. Bellelli G, Morandi A, Davis DH, et al. Validation of the 4AT, a new instrument for rapid delirium screening: a study in 234 hospitalised older people. Age Ageing 2014;43:496-502. 